\title{
Improvement in Lesion Detection with Whole-Body Oncologic Time-of-Flight PET
}

\author{
Georges El Fakhri*1, Suleman Surti ${ }^{* 2}$, Cathryn M. Trott ${ }^{1}$, Joshua Scheuermann², and Joel S. Karp² \\ ${ }^{1}$ Department of Radiology, Division of Nuclear Medicine and Molecular Imaging, Harvard Medical School and Massachusetts \\ General Hospital, Boston, Massachusetts; and ${ }^{2}$ Department of Radiology, University of Pennsylvania, Philadelphia, Pennsylvania
}

Time-of-flight (TOF) PET has great potential in whole-body oncologic applications, and recent work has demonstrated qualitatively in patient studies the improvement that can be achieved in lesion visibility. The aim of this work was to objectively quantify the improvement in lesion detectability that can be achieved in lung and liver lesions with whole-body ${ }^{18} \mathrm{~F}-$ FDG TOF PET in a cohort of 100 patients as a function of body mass index, lesion location and contrast, and scanning time. Methods: One hundred patients with BMls ranging from 16 to 45 were included in this study. Artificial $1-\mathrm{cm}$ spheric lesions were imaged separately in air at variable locations of each patient's lung and liver, appropriately attenuated, and incorporated in the patient list-mode data with 4 different lesion-tobackground contrast ranges. The fused studies with artificial lesion present or absent were reconstructed using a list-mode unrelaxed ordered-subsets expectation maximization with chronologically ordered subsets and a gaussian TOF kernel for TOF reconstruction. Conditions were compared on the basis of performance of a 3-channel Hotelling observer signal-tonoise ratio in detecting the presence of a sphere of unknown size on an anatomic background while modeling observer noise. Results: TOF PET yielded an improvement in lesion detection performance (3-channel Hotelling observer signal-to-noise ratio) over non-TOF PET of $8.3 \%$ in the liver and $15.1 \%$ in the lungs. The improvement in all lesions was $20.3 \%, 12.0 \%, 9.2 \%$, and $7.5 \%$ for mean contrast values of $2.0: 1,3.2: 1,4.4: 1$, and $5.7: 1$, respectively. Furthermore, this improvement was $9.8 \%$ in patients with a BMI of less than 30 and $11.1 \%$ in patients with a $\mathrm{BMI}$ of 30 or more. Performance plateaued faster as a function of number of iterations with TOF than non-TOF. Conclusion: Over all contrasts and body mass indexes, oncologic TOF PET yielded a significant improvement in lesion detection that was greater for lower lesion contrasts. This improvement was achieved without compromising other aspects of PET imaging.

Key Words: WB-18FDG; TOF-PET; lesion detection

J Nucl Med 2011; 52:347-353

DOI: 10.2967/jnumed.110.080382

\footnotetext{
Received Jun. 21, 2010; revision accepted Dec. 1, 2010.

For correspondence or reprints contact: Joel S. Karp, Department of Radiology, University of Pennsylvania School of Medicine, 423 Guardian Dr., Blockley Hall, Room 425, Philadelphia, PA 19104.

E-mail: joelkarp@mail.med.upenn.edu

${ }^{*}$ Contributed equally to this work.

COPYRIGHT @ 2011 by the Society of Nuclear Medicine, Inc.
}

$\mathbf{T}$ ime-of-flight (TOF) PET has great potential for the improvement of image quality in whole-body oncologic applications (1-6). Recent work by Karp et al. (7) has reported faster and more uniform convergence of lesion contrast and demonstrated qualitatively in phantoms and patient studies the improvement that can be achieved in subjective lesion detection. Recent work by Kadrmas et al. (8) on lesion detection in a physical phantom also found improvement in detection performance with TOF PET. However, little work has been done on the objective assessment of the benefits of TOF PET for lesion detection in clinical whole-body ${ }^{18} \mathrm{~F}$ FDG oncologic studies, partly because of the absence of a gold standard in patient studies and the difficulty of performing such a study on enough patients to ensure sufficient statistical power. Therefore, the aim of this work was to quantify the improvement of detectability of lung and liver lesions that can be achieved with TOF PET in whole-body ${ }^{18} \mathrm{~F}-\mathrm{FDG}$ studies using an approach previously developed in our laboratory that allows the incorporation of realistic lesions within lesion-absent whole-body ${ }^{18}$ F-FDG studies (9).

Actual whole-body patient data, rather than phantom data, were used in order to encompass a large range of realistic sizes and, therefore, activity and attenuation distributions. Our hypothesis was that the gain in performance of TOF imaging depends on patient size, count statistics, noise structure, and lesion contrast. Because these characteristics cannot be perfectly reproduced in phantom studies, we used patient data with synthetic lesions in order to capture the physiologic variability of ${ }^{18} \mathrm{~F}-\mathrm{FDG}$ uptake in the torso and abdomen.

\section{MATERIALS AND METHODS}

\section{Patient Studies}

One hundred patient studies were selected for this investigation. Each patient was scanned under the standard imaging protocol at the University of Pennsylvania Medical Center: a 555-MBq injection of ${ }^{18} \mathrm{~F}-\mathrm{FDG}$ followed by initiation of scanning $1 \mathrm{~h}$ afterward. The PET data were acquired in list-mode format with a 3-min scan time per bed position, providing the flexibility to reconstruct images for a 1-min scan time as well as $3 \mathrm{~min}$. A complete patient study typically involves $8-10$ overlapping bed positions. In this study, for each patient we selected a single bed position that was determined by experienced nuclear medicine physicians to have 
normal ${ }^{18} \mathrm{~F}$-FDG uptake in the torso region (including lungs and liver).

The body mass index (BMI) (weight $[\mathrm{kg}] / \mathrm{height}^{2}\left[\mathrm{~m}^{2}\right]$ ) as defined by the National Institutes of Health publications 045283 and 98-4083 (10) was used to quantify patient size. In our population, BMI ranged from 16 to 45.4 , distributed as follows: 41 bed positions were associated with patients with a normal BMI $(<24.9)$, 28 bed positions with overweight patients $(25<\mathrm{BMI}<$ 29.9), 27 bed positions with obese patients (30< BMI $<39.9$ ), and 5 bed positions with extremely obese patients (BMI $>40)$.

\section{Scanner and Image Reconstruction}

All data acquisitions and patient studies were performed on a Gemini TF PET/CT scanner (Philips) at the University of Pennsylvania. The Gemini TF is a TOF-capable, fully 3-dimensional PET scanner together with a 16-slice Brilliance CT scanner (11). The PET patient bore has a diameter of $71.70 \mathrm{~cm}$ with active transverse and axial fields of view of 57.6 and $17.98 \mathrm{~cm}$, respectively. The reconstructed spatial resolution is $4.8 \mathrm{~mm}$ (center of field of view), and the system energy resolution (in full width at half maximum) is $11.5 \%$ at $511 \mathrm{keV}$, which allows the default energy window to be set at $440-665 \mathrm{keV}$. The system timing resolution measured with a low-activity source is 585 ps (in full width at half maximum), although the clinical data presented here were acquired with a system timing resolution of 670 ps because of the effects of higher counting rates.

Images were reconstructed using a blob-based, list-mode iterative algorithm with and without TOF information (12). In this work, the data were arranged in 33 geometrically ordered subsets, and an unrelaxed $(\lambda=1)$ ordered-subsets expectation maximization update equation was used for reconstruction. Attenuation was corrected using the CT transmission data, and scatter was estimated using a recently developed TOF version of model-based single-scatter simulation that generates a distribution of scatter in both the radial and the time bins (13). Delayed coincidence window technique was used to estimate the random coincidences in the collected data. No postreconstruction smoothing filter was used (the reconstruction was blob-based). The image matrix size was $576 \times 576 \times 180 \mathrm{~mm}$, and the voxel size was $4 \times 4 \times 4 \mathrm{~mm}$.

\section{Lesion-Present Image Generation}

To create lesion-present clinical studies while ensuring perfect knowledge of the presence and location of each lesion (gold standard), 10-mm spheric lesions were added to disease-free bed positions, yielding fused lesion-present studies. Numeric simulation of lesions may lead to errors due to an incomplete modeling of scanner components. Therefore, we used $10-\mathrm{mm}$ plastic spheres that were filled with ${ }^{18} \mathrm{~F}$-FDG $(5-50 \mathrm{MBq} / \mathrm{mL})$ and acquired data for the spheres in air at specific locations within the scanner field of view that were chosen to overlap with regions within the lung and liver for each patient. The sphere data were acquired in list mode over 1-5 min of scan time such that at least 5 million counts were collected at each sphere position. For each patient, regions of interest were drawn in the liver and lung regions to measure the mean background count density $\left(\mathrm{C}_{\mathrm{B}}\right)$ in the fully corrected reconstructed image. To add a lesion with an uptake ratio $u$ to a patient, we then used $(u-1) \times C_{B}$ counts from a selected sphere dataset (extracted sphere list file). Because the sphere data are collected in air, they do not represent any attenuation effects, and so the extracted sphere list file was attenuated using the transmission map for the patient study. Finally, this lesion list file was then randomly mixed on an event-by-event basis with the patient list file to form a "fused" list file representing a patient with a $10-\mathrm{mm}$ lesion in the lung or liver scanned for $3 \mathrm{~min}$. The fused list file was then reconstructed using all corrections in the standard way for TOF and non-TOF reconstruction algorithms, for both 1- and 3-min scan times. We assumed that scatter associated with the sphere acquired in air was negligible as compared with the scatter associated with the patient. Therefore, the scatter estimate used for the fused datasets was the same as the one generated for no-lesion-present patient datasets and hence was done appropriately for all patient sizes. The lesion incorporation strategy can be explained as follows: the extracted sphere data with a fixed number of counts representing lesion contrast were attenuated using the patient transmission map to generate the attenuated sphere dataset, which was then added to the patient emission data to generate the fused dataset with an artificial lesion.

To validate the realism of the lesion generation strategy in TOF and non-TOF PET, a phantom study was undertaken. A cylindric phantom (diameter, $20 \mathrm{~cm}$ ) was filled with an approximately 17 $\mathrm{MBq} / \mathrm{mL}$ solution of ${ }^{18} \mathrm{~F}-\mathrm{FDG}$ and a first acquisition performed for $10 \mathrm{~min}$. Next, three 10 -mm-diameter spheres were filled with a solution of ${ }^{18} \mathrm{~F}$-FDG to achieve a sphere-to-background ratio of $2: 1$. The spheres were physically placed within the cylinder at $0^{\circ}$, $120^{\circ}$, and $240^{\circ}$, and a second acquisition was performed under identical conditions to the first acquisition. Finally, the spheres were mounted on the positioning grid at a location that corresponded to their location inside the cylindric phantom and acquired alone in air and then added to the cylinder scan (fused lesion-present study). This allowed us to compare profiles, as well as noise and sphere-to-background contrast ratios, in TOF and non-TOF PET.

\section{Lesion-Present and Lesion-Absent Conditions}

A major benefit of using a mathematic observer is the ability to explore a large number of conditions without being limited by the number of images that can be submitted to a human observer. In our study, we considered 6 conditions: The first condition was TOF or non-TOF PET. The second condition was a BMI of less than 30 (73 subjects) or of 30 or more (27 subjects), which represent the ranges for average light patients and average heavy patients, respectively. The third condition was lesion location in the liver or the lungs, which represent high and low ${ }^{18} \mathrm{~F}$-FDG uptake backgrounds, respectively. The fourth condition was lesion contrast and consisted of 4 contrast ranges for each lesion location-6.5:1, 5.0:1, 3.5:1, and 2.0:1 in the liver and 5.0:1, 4.0:1, 3.0:1, and 2.0:1 in the lung-leading to mean contrast ranges over both lesion locations of 5.7:1, 4.4:1, 3.2:1, and 2.0:1. The fifth condition was the acquisition time of $3 \mathrm{~min}$ or $1 \mathrm{~min}$, which represent the upper and lower limits of clinical scan times per bed position. The sixth condition was the number of iterations performed in the reconstruction $(1,2,3, \ldots 10)$. Therefore, the total number of lesion-present studies was $2($ TOF $) \times 100($ BMI $) \times 2$ (location) $\times 4$ (contrast $) \times 2($ time $) \times$ 10 (iterations) $=32,000$ studies. The total number of lesion-absent studies was $2(\mathrm{TOF}) \times 100(\mathrm{BMI}) \times 2$ (time) $\times 10$ (iterations) $=$ 4,000 studies. Therefore, the total number of studies considered was 36,000 .

\section{Channelized Hotelling Observer (CHO)}

Given the large number of studies considered in this work, we chose a numeric observer, as opposed to a human observer, to assess lesion detection SNR. The acquisition and processing schemes were assessed on the basis of performance of a model observer in detecting the presence of a spheric lesion of unknown size on an 
anatomic background. The model observer was a 3-dimensional, 3-channel CHO (14), by which the $15 \times 15 \times 5$ pixel subimage data were processed through the frequency channels that are believed to exist in the human visual system. The $15 \times 15 \times 5$ volume contained the lesion at the center of the image (only 1 lesion was present in any volume). The CHO signal-to-noise ratio (SNR) is given by:

$$
S N R_{C H O}^{2}=(\Delta f)^{t} S_{2}^{-1}(\Delta f),
$$

Eq. 1

where $\Delta f$ is the mean interclass channel output difference vector, and $S_{2}$ is the intraclass scatter matrix, calculated from the (channelized) covariance matrices $M_{1}$ and $M_{2}$ of the 2 classes (lesionpresent and lesion-absent), being discriminated by $S_{2}=\frac{M_{1}+M_{2}}{2}$. We used a 3-channel difference-of-gaussians $\mathrm{CHO}$ (14) with (radially symmetric) channel profiles $C_{0}, C_{1}$, and $C_{2}$ given by:

$$
C_{j}(\rho)=e^{-\frac{1}{2}\left(\frac{\rho}{2 \sigma_{j}}\right)^{2}}-e^{-\frac{1}{2}\left(\frac{\rho}{\sigma_{j}}\right)^{2}},
$$

Eq. 2

where $\rho$ is the spatial frequency, $j=1,3$ indexes the channels, $\sigma_{j}=$ $\sigma_{0} 2^{j-1}$, and $\sigma_{0}=0.052$. The parameters of this observer model are appropriate for a viewing distance of $60 \mathrm{~cm}$ and a displayed pixel size of $0.51 \mathrm{~mm}$; the effect of the observer noise on task performance was incorporated by doubling the diagonal elements of the channel covariance matrix (14). The lesion detectability performance estimated with the CHO SNR, $d_{A}$, can be related to the corresponding area under the receiving operator characteristic curve $\left(A_{z}\right)$ by:

$$
d_{A}=2 \operatorname{erf}^{-1}\left(2 A_{z}-1\right),
$$

where $e r f^{-1}$ is the inverse error function. The lesion present in the lungs (or liver) and background volumes were used to compute the 3-dimensional CHO SNR for lesion detection in the lung (or liver), in each $15 \times 15 \times 5$ subvolume. The error bars were computed using propagation of errors on detectability as previously proposed by Abbey et al. (15).

\section{RESULTS}

\section{Validation of Image Generation}

Figure 1 shows the results of the validation for generating lesion-present studies in the cylindric phantom. The top row shows a selected slice containing all 3 spheres physically inside the cylinder, and the bottom row shows the sphere data added mathematically to the cylinder (fused data). Good agreement is observed between the 2 reconstructed slices for both TOF and non-TOF PET. The corresponding profiles with the lesion physically present and mathematically added to the background also agree well with each other for both TOF and non-TOF PET. The pixel noise in the background in the fused lesion-present study was $6.2 \%$ in TOF PET, as compared with $6.1 \%$ in the physical lesionpresent TOF PET. For non-TOF PET, the pixel noise was $6.5 \%$ and $6.3 \%$, respectively. The sphere-to-background contrast recovery coefficient was $25.0 \% \pm 2.6 \%$ in the fused lesion-present TOF study, as compared with $22.5 \% \pm 1.6 \%$ in the physical lesion-present study. These values were $24.0 \% \pm 1.8 \%$ and $24.9 \% \pm 1.5 \%$, respectively, for nonTOF PET. As expected from previous studies for a $20-\mathrm{cm}-$ diameter cylinder, no significant gain in contrast recovery was observed with TOF imaging (7). Note that all sphereto-background contrast ratios were consistent with the actual 2:1 ratio used when filling the phantom.

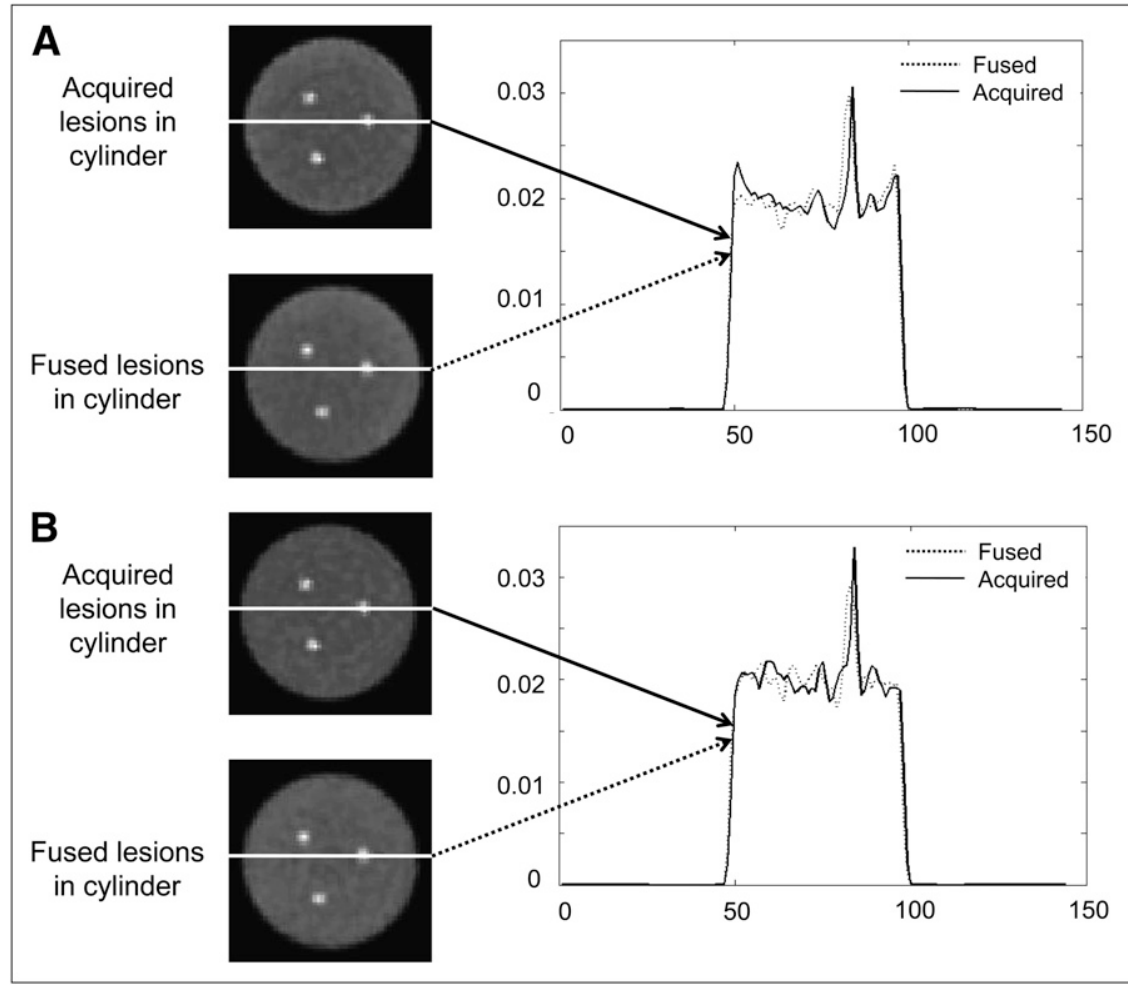

FIGURE 1. Transverse slices of reconstructed TOF (A) and non-TOF (B) PET images of cylindric phantom acquired with spheres physically inside cylinder, as well as with sphere data added to uniform cylinder without sphere (fused data), along with corresponding profiles through acquired and added spheres. 


\section{Improvement of Lesion Detection with TOF PET}

Figure 2 shows representative transverse slices of a lung lesion-present study with 3.5:1 contrast in a patient with a normal BMI of 19 and a liver lesion-present study with 2:1 contrast in a patient with a high BMI of 42 . Qualitatively, the lung lesion is hard to detect in the non-TOF PET study but is more easily discernable in the TOF PET study. Although the liver lesion is detectable in both non-TOF and TOF PET studies, lesion contrast was greater and background liver noise lower on TOF PET than on non-TOF PET.

Lesion Detection as Function of Lesion Location. Figure 3 shows the performance in lesion detection for TOF PET and non-TOF PET as a function of iteration number in the liver and lung lesions. The results are averaged over all BMIs, contrast levels, and scan times. TOF PET performance converged faster (at an earlier iteration number, e.g., iteration 4) than non-TOF (e.g., iteration 4) in the liver and especially the lung. Furthermore, lesion detection SNR was significantly higher for all iterations for TOF than for nonTOF, both in the lung $(P<0.01)$ and in the liver $(P<$ $0.01)$. The ratio of liver lesion SNR with TOF PET, as compared with non-TOF PET, varied from $17 \%$ in iteration 1 to $5 \%$ in iteration 10 . The same ratio in lung lesions varied from $22 \%$ in iteration 1 to $9 \%$ in iteration 10 .

Lesion Detection as Function of Scan Time. Figure 4 shows the improvement in lesion detection achieved with TOF PET as compared with non-TOF PET for scans of 3 and $1 \mathrm{~min}$ per bed position as a function of iteration number. The results are averaged over all BMIs, contrast levels, and lesion locations. The ratio of lesion detection SNR is

Non-TOF PET

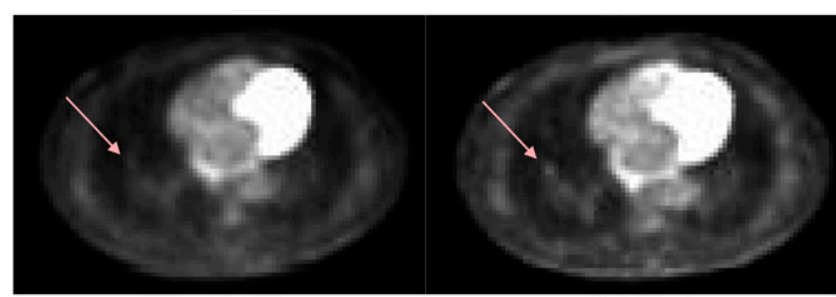

Lung lesion (3.5:1), $\mathrm{BMI}=19$

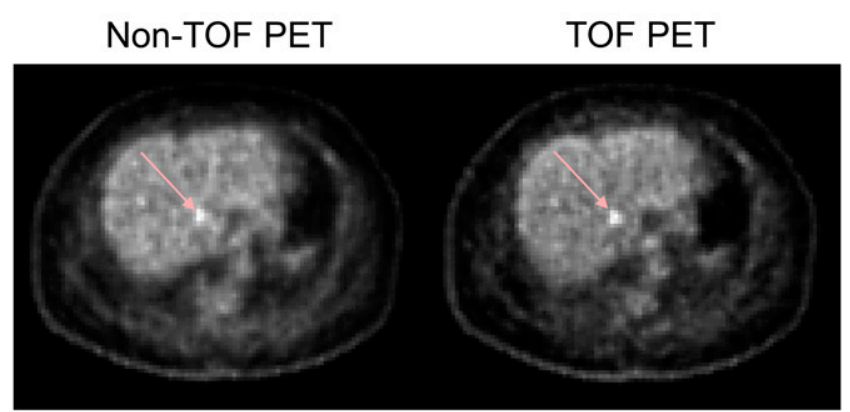

Liver lesion (2:1), BMI $=42$

FIGURE 2. Transverse slices showing lung lesion (arrows) with 3.5:1 contrast in patient with normal BMI of 19 (top) and liver lesion (arrows) with 2:1 contrast in patient with high BMI of 42 (bottom).

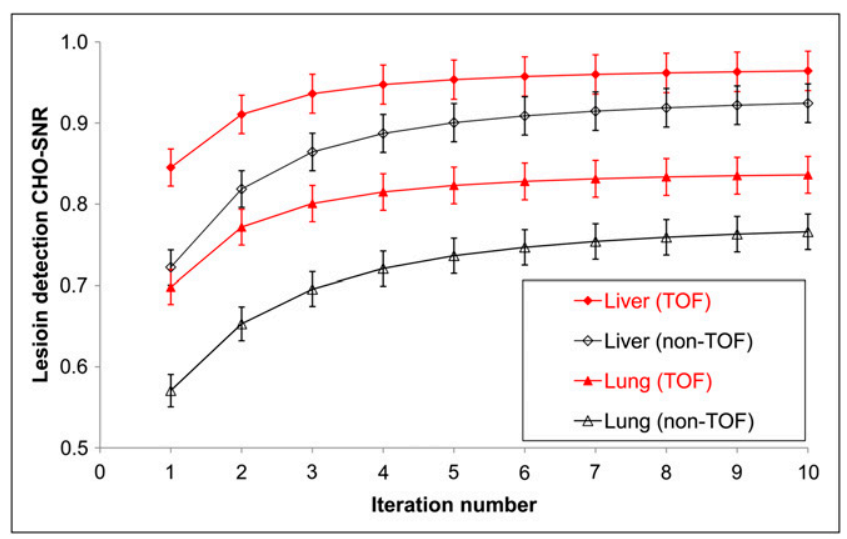

FIGURE 3. TOF and non-TOF PET CHO SNR computed for lung and liver lesions over all lesion contrasts and subject BMls as function of iteration number.

always greater than 1 . The greatest improvement in lesion detection with TOF as compared with non-TOF PET was at low iteration numbers. Furthermore, the improvement in lesion detectability with TOF as compared with non-TOF PET was greater for 1-min scans than for 3-min scans. This finding is consistent with the fact that a greater gain in performance is achieved with TOF at low SNRs.

Lesion Detection as Function of Lesion Contrast. Figure 5 shows the gain in lesion detection with TOF as compared with non-TOF PET as a function of lesion contrast and iteration number. The results are averaged over all BMIs, scan times, and lesion locations. As expected, lesion detectability was always better with TOF than with non-TOF (ratio always $>1$ ). More interestingly, the greatest gain in performance was achieved at the lowest lesion contrast (i.e., 2.0:1) and the smallest gain in performance at the highest lesion contrast (i.e., 5.7:1).

Lesion Detection as Function of BMI. Figure 6 shows the gain in lesion detection with TOF as compared with non-

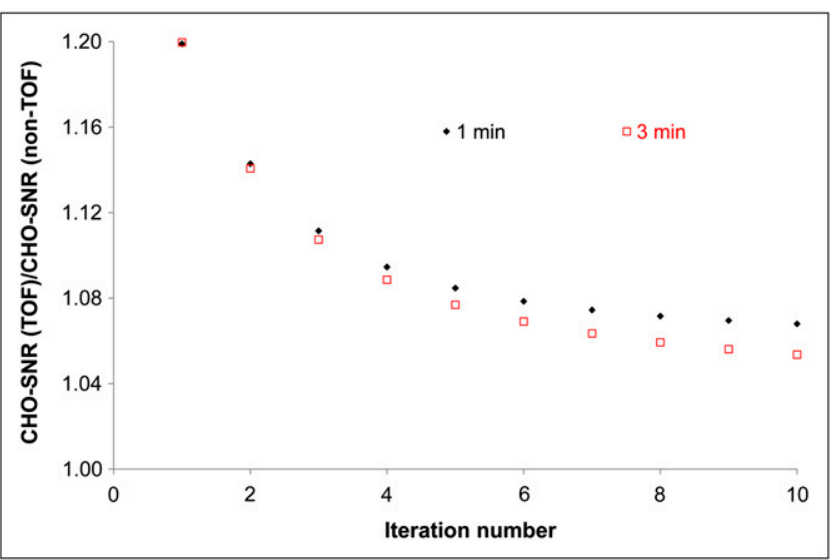

FIGURE 4. Ratio of TOF to non-TOF PET CHO SNR computed over all lesion locations; mean contrast values of 2.0:1, 3.2:1, and $4.4: 1$; and patient BMls as function of iteration number for acquisitions of 1 and 3 min per bed position. 


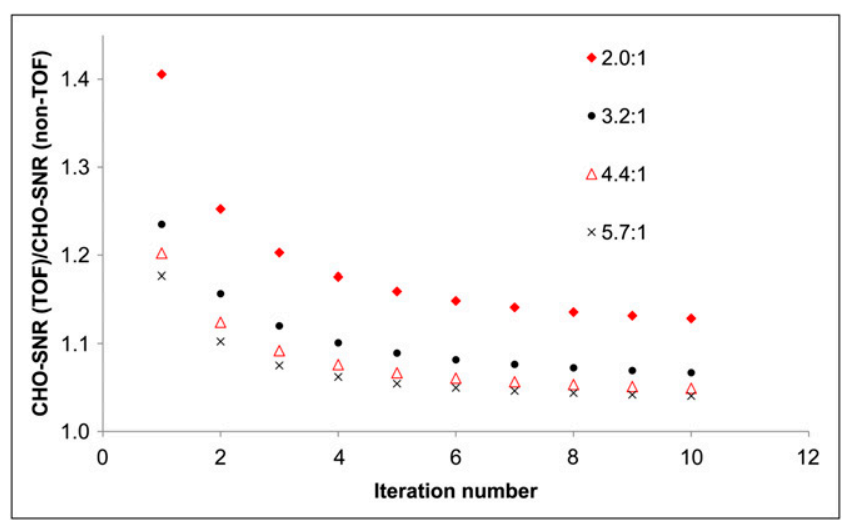

FIGURE 5. Ratio of TOF to non-TOF PET CHO SNR computed over all lesion locations and patient BMls as function of iteration number for different lesion-to-background contrasts.

TOF PET as a function of patient BMI and iteration number. The results are averaged over all scan times and lesion contrasts and locations. Again, the greatest gain in performance is achieved for a BMI of 30 or more; that is, TOF yielded the best improvement in large patients when lesion detection was the most challenging.

Gain in Lesion Detection Due to TOF PET. Figure 7 summarizes the gain in lesion detection as measured by the CHO SNR, computed over lesion contrasts, subject BMIs, and locations. All results are shown after 3 iterations of image reconstruction, which correspond to the number of iterations used clinically with TOF PET at our institution. There was a significant improvement in all lesions (over all BMIs, lesion locations, and scan times) of $20.3 \%, 12.0 \%$, $9.2 \%$, and $7.5 \%$ for mean contrast of $2.0: 1,3.2: 1,4.4: 1$, and 5.7:1, respectively $(P<0.01)$. When all lesion contrasts were pooled, this improvement was also significant and was $8.3 \%$ in liver tumors and $15.1 \%$ in lung tumors $(P<0.01)$. Finally, the improvement in lesion SNR detectability was $9.8 \%$ in patients with a BMI of less than 30 and $11.1 \%$ in patients with a BMI of 30 or more $(P<0.01)$.

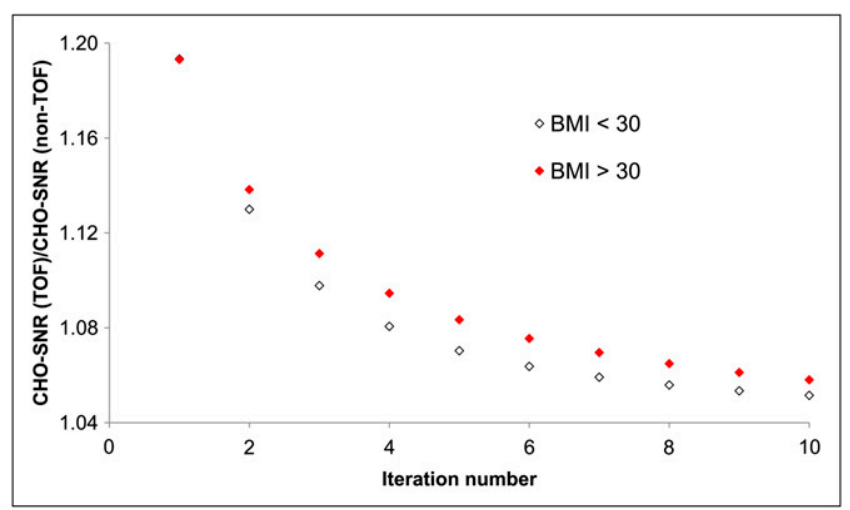

FIGURE 6. Performance of TOF and non-TOF PET for lesion detection as function of patient $\mathrm{BMI}$ and iteration number. Results correspond to ratio of TOF to non-TOF PET CHO SNR and are averaged over all scan times and lesion contrasts and locations.

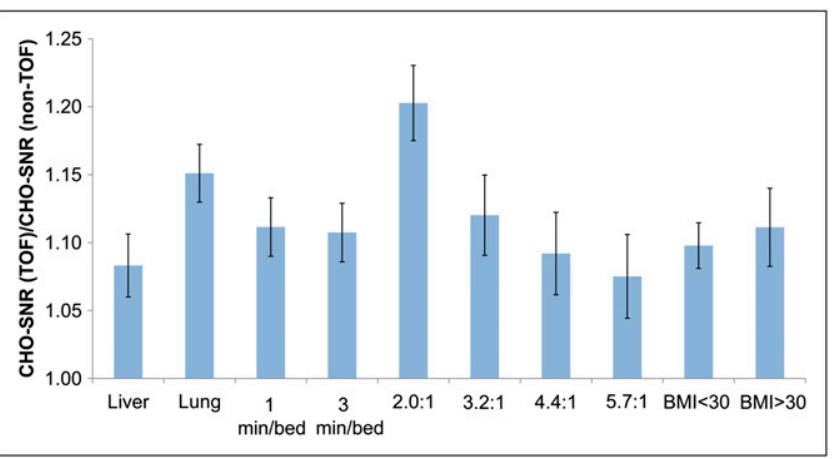

FIGURE 7. Ratio of TOF to non-TOF PET CHO SNR computed over all lesion contrasts and locations as well as BMls for $n=3$ iterations.

\section{DISCUSSION}

We have determined the gain in performance that can be achieved when TOF PET is applied in whole-body ${ }^{18} \mathrm{~F}$-FDG PET using a task-based metric, that is, lesion detection as measured by a CHO. Our approach has the advantage of using clinically realistic images with knowledge of whether lesions are present or absent. The realistic process of lesion incorporation into normal whole-body TOF PET ${ }^{18} \mathrm{~F}-\mathrm{FDG}$ studies has yielded a valuable set of lesion-present and lesion-absent studies in 100 patients (i.e., 36,000 studies). A subset of this dataset will be used in the future to confirm the results in a reduced number of conditions using human observers. Because the aim of this work was to assess the detection of lesions and not the determination of lesion shape or density, we considered a model consisting of $1-\mathrm{cm}$ spheric lesions. This is a reasonable model for small metastatic lesions as seen routinely in lung and liver cancers. Largerlesion models would need to take into account possible necrosis, which was not modeled in our study. We have validated in this work and previous work (9) our methodology for lesion insertion in physical phantoms. The use of the same scatter file for lesion and no-lesion data is justified.

In contrast with previously published studies that reported comparison of TOF and non-TOF PET in physical phantoms $(7,8)$, this study included a population of patients with a large range of BMIs and lesion contrasts to make possible the assessment of performance of TOF PET as a function of patient size, acquisition time, lesion location, and contrast and reconstruction iteration number. Furthermore, the use of clinical data, in contrast to numerically simulated data, ensured that all physical factors, such as scatter, attenuation, randoms, and dead time, were correctly included for all body sizes and physiologic activity distributions. Thus, we are confident that conclusions drawn from the data can be extrapolated to the clinical setting after confirmation.

Our results show several interesting and important features. As expected in clinical studies, the covariance in the images is dominated by variability in patient backgrounds, rather than statistical noise. Hence, the images acquired for 
3 min per bed position showed mildly improved performance compared with those acquired for $1 \mathrm{~min}$, as the only difference between these studies was statistical noise (same background viability). Also as expected, the improvement in the SNR of TOF over non-TOF PET was greatest for lower-contrast lesions. High-contrast lesions are more easily detectable, yielding little improvement by using TOF. Another feature of our results is that liver lesions showed higher detectability than lung lesions, because of the higher number of counts in liver background than in lung background and therefore lower noise in liver lesions than in lung lesions (e.g., a 4:1 liver lesion has higher counts than a 4:1 lung lesion because of the higher background activity of liver than lung). In addition, lung lesions showed greater improvement in performance with TOF PET than did liver lesions, because of the lower overall detectability of lung lesions. Our results were also interesting in showing that TOF detectability converged at an earlier iteration number than did non-TOF detectability and therefore produced high-detection-performance images at a lower noise level. A final important feature of our results was the greater improvement from non-TOF to TOF PET found for highBMI patients than for low BMI patients, suggesting that TOF PET is most beneficial for imaging larger patients.

Pooling liver and lung lesions in the results can lead to confusing comparisons. Lung lesions with the same contrast ratio as liver lesions necessarily have higher noise because of the lower background counts in the lungs. Therefore, relative SNRs between the 2 populations are not easily comparable. Rather, we focused on the performance improvement for a given lesion type (ratio of SNRs for TOF and non-TOF) and the relative performance improvement for different lesion types (comparison of ratios).

Our results are consistent with our previous work (7), for which we reported faster and more uniform convergence of lesion contrast and demonstrated qualitatively in phantoms and patient studies the improvement that can be achieved in subjective lesion detection. Furthermore, our results show that the greatest gains in image quality in terms of lesion detectability were achieved for the lowest lesion contrast of 2.0:1 ( 20\%) and in the largest subjects. This is a significant gain that can be crucial in marginally detectable tumors such as the lung lesion shown in Figure 2. Our results are also consistent with recent work that found improvement in detection in a physical phantom with TOF PET (8). Furthermore, our results suggest that the gain achieved with TOF PET can be used in several ways. One possibility is to take advantage of the gain achieved with TOF PET to reduce scanning time, therefore increasing patient comfort and minimizing patient motion. Another interesting possibility is to reduce the injected dose and therefore reduce the exposure of patients and health professionals to radiation. The improvement in lesion detection with TOF PET over non-TOF was achieved while keeping all acquisition parameters constant (e.g., injected dose, acquisition time, acquisition geometry, and the lower and upper limits of detectability).
The task considered in this work was lesion detection, measured by CHO SNR $(14,16,17)$. Although lesion detection performance is best assessed using human observer studies $(8,18,19)$, the numeric observer approach allows more rapid assessment of a large number of conditions (e.g., scanning time, lesion contrast, patient BMI, and lesion location) that can result in a large number of images (e.g., 30,000)-more than can be practical for human observer studies. Furthermore, the rank-order-assessing performance is usually preserved between the channelized observer and human observer studies. The next step of this research will be to design focused human observer studies to confirm the main findings obtained with a channelized observer in the present work.

\section{CONCLUSION}

We have determined the improvement that can be achieved with TOF PET over non-TOF PET under identical realistic conditions in a cohort of 100 patients. TOF PET yielded a significant improvement in lesion detection in oncologic studies over all contrasts and BMIs, and this improvement was greater for lower lesion contrasts. Furthermore, the greatest gains achieved with TOF PET were for the shortest-acquisition studies (1 min per bed position) and in the largest subjects (BMI $\geq 30$ ), confirming that TOF PET could play a key role in detecting marginally detectable lesions without compromising other aspects of PET imaging.

\section{ACKNOWLEDGMENTS}

This work was supported in part by grants RO1CA113941, R01-EB009056, EB005876, and R21-CA134812 from the National Institutes of Health (NIH). The contents of this paper are solely the responsibility of the authors and do not represent the official views of the NIH.

\section{REFERENCES}

1. Budinger TF. Time-of-flight positron emission tomography: status relative to conventional PET. J Nucl Med. 1983;24:73-78.

2. Tomitani T. A deconvolution function for single photon emission computed tomography with constant attenuation. IEEE Trans Nucl Sci. 1986;33:505-510.

3. Conti M, Bendriem B, Casey M, et al. First experimental results of time-of-flight reconstruction on an LSO PET scanner. Phys Med Biol. 2005;50:4507-4526.

4. Surti S, Karp JS, Popescu LA, Daube-Witherspoon ME, Werner M. Investigation of time-of-flight benefit for fully 3-D PET. IEEE Trans Med Imaging. 2006;25: 529-538.

5. Surti S, Karp JS. Experimental evaluation of a simple lesion detection task with time-of-flight PET. Phys Med Biol. 2009;54:373-384.

6. Lois C, Jakoby BW, Long MJ, et al. An assessment of the impact of incorporating time-of-flight information into clinical PET/CT imaging. J Nucl Med. 2010; 51:237-245

7. Karp JS, Surti S, Daube-Witherspoon ME, Muehhlehner G. The benefit of timeof-flight in PET imaging: experimental and clinical results. J Nucl Med. 2008; 49:462-470

8. Kadrmas DJ, Casey ME, Black NF, Hamill JJ, Panin VY, Conti M. Experimental comparison of lesion detectability for four fully-3D PET reconstruction schemes. IEEE Trans Med Imaging. 2009;28:523-534.

9. El Fakhri G, Santos PA, Badawi RD, Holdsworth CH, Van den Abbeele AD, Kijewski MF. Impact of acquisition geometry, image processing, and patient size on lesion detection in whole-body FDG-PET. J Nucl Med. 2007;48:1951-1960.

10. Clinical Guidelines on the Identification, Evaluation, and Treatment of Overweight and Obesity in Adults: The Evidence Report. Bethesda, MD: The National Institutes of Health; 1998. NIH Publication 98-4083. 
11. Surti S, Kuhn A, Werner ME, Perkins AE, Kolthammer J, Karp JS. Performance of Philips Gemini TF PET/CT scanner with special consideration for its time-offlight imaging capabilities. J Nucl Med. 2007;48:471-480.

12. Popescu LM. Iterative image reconstruction using geometrically ordered subsets with list-mode data. Paper presented at: IEEE Nuclear Science Symposium and Medical Imaging Conference, 2004; Rome, Italy.

13. Werner ME, Surti S, Karp JS. Implementation and evaluation of a 3D PET single scatter simulation with TOF modelling. Paper presented at: IEEE Nuclear Science and Medical Imaging Conference, 2006; San Diego, CA.

14. Abbey CK, Barrett HH. Human- and model-observer performance in rampspectrum noise: effects of regularization and object variability. J Opt Soc Am A Opt Image Sci Vis. 2001;18:473-488.
15. Abbey CK, Barrett HH, Eckstein MP. Practical issues and methodology in assessment of image quality using model observers. Proc SPIE. 1997;3032:182-194.

16. Fiete RD, Barrett HH, Smith WE, Myers KJ. Hotelling trace criterion and its correlation with human-observer performance. J Opt Soc Am A. 1987;4:945-953.

17. Yao J, Barrett HH. Predicting human performance by a channelized Hotelling observer model. Proc SPIE. 1992;1768:161-168.

18. Farquhar TH, Llacer J, Hoh CK, et al. ROC and localization ROC analyses of lesion detection in whole-body FDG PET: effects of acquisition mode, attenuation correction and reconstruction algorithm. J Nucl Med. 1999;40:2043-2052.

19. Lartizien C, Kinahan PE, Comtat C. A lesion detection observer study comparing 2-dimensional versus fully 3-dimensional whole-body PET imaging protocols. J Nucl Med. 2004;45:714-723. 